\title{
Systematic reviews and guidelines for oral complications of cancer therapies: current challenges and future opportunities
}

\author{
Michael T. Brennan • Fred K. L. Spijkervet • \\ Linda S. Elting
}

Received: 15 October 2009 / Accepted: 23 February 2010/Published online: 10 March 2010

(C) Springer-Verlag 2010

The annual incidence of cancer in the world is estimated at more than 11 million cases with at least 1.3 million in the United States of America [1,2]. Earlier cancer detection and advances in cancer therapies have provided important management advances to improve survival and quality of life. Common cancer treatment strategies include surgical resection, chemotherapy, radiotherapy, and hematopoietic stem cell transplantation. The goal of such treatments is to cure the patient from the cancer. However, side effects from these therapies can limit the effectiveness of treatment and have a marked impact on the patient's quality of life both during and after completion of the cancer therapy. The oral cavity is a common site of complications related to cancer therapies. The Surgeon General's report on Oral Health in America estimates that more than $30-35 \% \quad(400,000$ annually) of patients undergoing cancer treatment will develop oral complications [3].

The Surgeon General's report-Oral Health in Americacalls for both the building of the science and evidence base and the application of science effectively to improve oral

M. T. Brennan $(\triangle)$

Department of Oral Medicine, Carolinas Medical Center,

P.O. Box 32861, Charlotte, NC 28203, USA

e-mail: mike.brennan@carolinas.org

F. K. L. Spijkervet

Department of Oral and Maxillofacial Surgery,

University Medical Center Groningen, University of Groningen,

P.O. Box 30.001, Groningen 9700 RB, The Netherlands

e-mail: f.k.l.spijkervet@kchir.umcg.nl

L. S. Elting

Department of Biostatistics,

The University of Texas M.D. Anderson Cancer Center,

1515 Holcombe Blvd,

Houston, TX 77030, USA

e-mail: lelting@mdanderson.org health. This recommendation development process aims to effectively transfer research findings in the field of oncology, including oral complications seen with cancer therapies and oral management prior to the start of cancer therapy, to the public and health professionals. Well developed, evidencebased management recommendations have the potential to enhance the appropriateness of clinical practice, improve the quality of oral health care, lead to better patient outcomes, improve cost-effectiveness, and identify areas of further research needs. In order to effectively change perceptions of the burden of illness of oral complications from cancer therapies, a complete understanding of the impact of these complications is vital. Concern for underestimating the impact of oral complications may result in avoidance or delay in appropriate care for cancer patients.

In order to establish recommendations for pre-, interim-, and post-cancer therapy management of oral problems, an understanding of the scope of oral complications from cancer therapy must be established. However, the literature reports a wide range of oral complications with varying incidences due to study flaws, definitions, and grading scales. This lack of clarity is reflected in a lack of comprehensive and effective oral management regimens in the clinical arena. There is a pressing need for a systematic review of this large body of world literature to establish the nature and incidence of oral complications for each cancer therapy. With a deeper understanding of the epidemiology, assessment of oral care regimens to minimize such complications can be appropriately formulated and evaluated.

The many volunteers from the Oral Care Study Group of the Multinational Association of Supportive Care in Cancer and International Society of Oral Oncology (MASCC/ ISOO) have completed systematic reviews and guideline recommendations of the most common oral complications 
of cancer therapy, which are included in the following systematic reviews from the Oral Care Study Group of MASSC/ISOO. Not included in the current monograph is a review of oral mucositis. The Mucositis Study Group of the MASCC/ISOO has held two Consensus Conferences regarding the impact and treatment of cancer-related oral mucositis [4-6].

The first aim of the current systematic reviews was to determine the incidence or prevalence of the various oral complications by cancer therapy. While possible for oral complications such as xerostomia and oral pain, the lack of sufficient data made this goal challenging for many of the other oral complications. Additionally, while certain studies assessed oral complications from newer emerging cancer therapies, a systematic evaluation was of limited value due to lack of data.

The next aim of the current reviews was to identify the effect of each oral complication on quality of life (QOL) measures and economic impact. Numerous QOL measures are available with some questions directed at xerostomia, oral pain, and dysgeusia. Otherwise, QOL was infrequently assessed in relation to other oral complications. Very limited data was available for all oral complications regarding economic impact.

The final aim was to provide guideline recommendations for the management of the various oral complications. Again, a large variation in well-designed randomized controlled trials for the different oral complications was apparent with level 1 evidence available for a few oral complications, but more commonly level 2, 3, or 4 evidence was present to base guideline recommendations.

Another challenge for the present systematic reviews was interpreting the wide variation in outcome measures for the different oral complications. Clear definitions of each oral complication were frequently lacking and different measures to assess severity grade were identified. Comparisons between the different Likert scale and visual analog scales were not possible. A more uniform approach to measuring the different oral complications would provide a clear advantage for comparison and treatment responses across various studies.

Clearly, large multicenter observational studies will provide much needed data for incidence/prevalence, severity, impact on QOL, and economic impact for different oral complications from cancer therapies. With a more thorough understanding on the burden of illness for the different oral complications from cancer therapies, more targeted preventive and management strategies can be sought.

Importantly, oral complications will often appear concurrently. For instance, a cancer patient may experience oral pain, mucositis, xerostomia, dysgeusia, and a fungal infection at the same time. A clearer understanding of the temporal relationship and the impact of a cluster of oral complications needs to be further explored.

The success of these reviews by the members of the Oral Care Study Group of MASCC/ISOO has provided the opening for future studies to explore these gaps and opportunities to enhance our understanding of the complicated interactions of the various oral complications.

Conflict of interest statement None to declare.

\section{References}

1. U.S. Cancer Statistics Working Group. United States cancer statistics: 2001 incidence and mortality. Atlanta, GA: Department of Health and Human Services, Centers for Disease Control and Prevention and National Cancer Institute (2004), pp 1-699

2. (2005) World Health Organization: Cancer [cited Sep 26]:. Available from http://www.who.int/cancer/en/.

3. U.S. Department of Health and Human Services. Oral Health in America: A Report of the Surgeon General, Executive Summary (2000) Rockville. U.S. Department of Health and Human Services, National Institute of Dental and Craniofacial Research, National Institutes of Health, MD

4. Sonis ST, Elting LS, Keefe D, Peterson DE, Schubert M, Hauer-Jensen M, Bekele BN, Raber-Durlacher J, Donnelly JP, Rubenstein EB, for the Mucositis Study Section of the Multinational Association of Supportive Care in Cancer and the International Society for Oral Oncology (2004) Perspectives on cancer therapy-induced mucosal injury: pathogenesis, measurement, epidemiology, and consequences for patients. Cancer 100:1995-2025

5. Rubenstein EB, Peterson DE, Schubert M, Keefe D, McGuire D, Epstein J, Elting LS, Fox PC, Cooksley C, Sonis ST, for the Mucositis Study Section of the Multinational Association of Supportive Care in Cancer and the International Society for Oral Oncology (2004) Clinical practice guidelines for the prevention and treatment of cancer therapy-induced oral and gastrointestinal mucositis. Cancer 100:2026-2046

6. Spielberger R, Stiff P, Bensinger W, Gentile T, Weisdorf D, Kewalramani T, Shea T, Yanovich S, Hansen K, Noga S, McCarty J, LeMaistre CF, Sung EC, Blazar BR, Elhardt D, Chen M, Emmanouilides C (2004) Palifermin for oral mucositis after intensive therapy for hematologic cancers. N Engl J Med 351:2590-2598 